\title{
Role of oral ondansetron for prevention of postoperative nausea and vomiting in laparoscopy assisted gynaecological surgery - a comparative study
}

Montosh Kumar Mondal ${ }^{*}$, Shahadat Hossain ${ }^{2}$, Beauty Rani Roy ${ }^{3}$, Begum Maksuda Farida Akhtar ${ }^{4}$, Joysree Roy ${ }^{5}$, AKM Akhtaruzzaman ${ }^{6}$

\begin{abstract}
${ }^{1}$ Assistant Professor, Department of Anaesthesiology, National Institute of Cancer Research \& Hospital (NICRH), Mohakhali, Dhaka, Bangladesh, ${ }^{2}$ Consultant Anaesthesiologist, Department of Anaesthesiology, Dhaka Dental College, Dhaka, ${ }^{3}$ Consultant, Department of Obstetrics and Gynaecology, OGSB Hospital and Institute of Reproductive and Child Health, Mirpur, Dhaka, Bangladesh ${ }^{4} \mathrm{MS}$ (Gynae and Obs) thesis part student, Department of Obstetrics and Gynaecology, BSMMU, Shahbag, Dhaka-1000, Bangladesh ${ }^{5}$ Consultant, Department of Obstetrics and Gynaecology, Dhaka Medical College, Dhaka1000, Bangladesh ${ }^{6}$ Associate Professor, Department of Anaesthesia, Analgesia and Intensive Care Medicine, BSMMU, Shahbag, Dhaka-1000, Bangladesh

*Corresponding author: Dr. Montosh Kumar Mondal, Assistant Professor, Department of Anaesthesiology, National Institute of Cancer Research \& Hospital (NICRH), Mohakhali, Dhaka, Bangladesh
\end{abstract}

\begin{abstract}
:
This study was undertaken to find out the efficacy of oral premedication with ondansetron to prevent post-operative nausea and vomiting in diagnostic gynaecological laparoscopy assisted surgery and to compare it with metoclopramide.

We studied fifty patients of ASA physical status I \& II, aged between 18-30 years and body weight between 50-60 kgs. The patients were randomized in equal numbers into two groups; Group A patients were received Tab Metoclopramide $10 \mathrm{mg}$ orally an hour before operation and regarded as control and Group $B$ patients were received Tab Ondansetron $(0.15 \mathrm{mg} / \mathrm{kg}$ ) or total $8 \mathrm{mg}$ orally an hour before operation as case. They received a standard general anesthetic. Post-operative analgesia was provided with per rectal diclofenac sodium (50mg). In the recovery room occurrences of nausea and vomiting was assessed for 24 hours. The incidence of nausea was $80 \%$ in Group-A, $24 \%$ in Group-B $(p<0.001)$ and vomiting was $64 \%$ in Group-A, $16 \%$ in Group-B $(p<0.001)$. The difference among the groups was statistically significant.
\end{abstract}

Key words: Laparoscopy assisted gynaecological surgery, PONV, oral ondansetron, metoclopramide.

(Journal of BSA, 2008; 21(2): 67-71)

\section{Introduction}

Postoperative nausea and vomiting (PONV) are among the most common postoperative complaints. Patients undergoing laparoscopy assisted surgery are at high risk for PONV. These are frequently the case of great distress to patients and it is often the worst memory of their hospital stay. ${ }^{1}$ The consequences of prolonged PONV range from unexpected admission of day case surgical patients, to physical, metabolic and psychological effects on the patients which slow their recovery and reduce their confidence in future surgery and anaesthesia. Persistent nausea and vomiting may result in dehydration, electrolyte imbalance and delayed discharge. Persistent retching or vomiting can cause tension on suture lines, venous hypertension and increased bleeding under skin flaps and can expose the subject to an increased risk of pulmonary aspiration of vomitus if airway reflexes are depressed from the residual effects of anaesthetic and analgesic drugs.

The incidence and severity of PONV has been decreasing over the last 10 years, due to the identification of precipitating factors, the use of better anaesthetics and preoperative medications, and improvement in operative techniques. ${ }^{3}$ Despite these changes, there is still an unacceptable frequency of PONV with incidences of up to $85 \%$ reported in some studies. ${ }^{4}$ Watcha \& White suggested that the incidence of $\mathrm{PONV}$ has remained fairly constant for decades with $20-30 \%$ of patients suffering from these unpleasant side-effects. ${ }^{5}$ 
Many drugs have so far been tried to prevent or alleviate this problem. The antiemetics that are currently being used for treatment in our country are prochlorperazine, metoclopramide and promethazine. Antiemetic such as metoclopramide is often used in the control of PONV. It acts peripherally as a cholinomimetic and centrally as a dopaminergic antagonist by enhancing the stimulatory effects of acetylcholine on intestinal smooth muscle, metoclopramide increase lower esophageal sphincter tone, speeds gastric empting, and lowers gastric fluid volume. It also produces an antiemetic effect by blocking dopaminergic receptors in the chemoreceptor trigger zone of the central nervous system. But most of the drugs are associated with undesirable side effects including sedation, hypotension, extrapyramidal symptoms, dysphoria, nervousness and may also cause delayed recovery from anaesthesia. ${ }^{6}$

Ondansetron is a selective competitive $5-\mathrm{HT}_{3}$ receptor antagonist with little or no effect on dopaminergic receptors. ${ }^{7}, 8$. It has a good safety profile. It does not appear to cause sedation, extrapyramidal signs or respiratory depression. The most common reported side effect is headache. ${ }^{9}$

Antiemetic prophylaxis may be justified in patients who are at greater risk of developing postoperative nausea and /or vomiting ${ }^{5}$ these include patients with a history of previous postoperative emesis, in day case surgical patient and women undergoing gynaecological procedure especially by laparoscopy.

In this study, we have investigated the efficacy of oral ondansetron to prevent PONV in diagnostic laparoscopy assisted gynaecological surgery and to compare it with oral metoclopramide.

\section{Materials and Methods}

Fifty female patients of ASA physical status I and II, age between 18-30 years and body weight between $50-60 \mathrm{~kg}$, scheduled to undergo diagnostic laparoscopy assisted gynecological surgery under general anaesthesia were studied. The study protocol was approved by institutional ethical committee of Bangabandhu Sheikh Mujib Medical University (BSMMU). Informed written consent was taken from the patient. They were randomly divided into two groups, twenty five patients in each. The randomization was done by blind envelop methods. Patients with persisting vomiting, received any antiemetic within 24 hours before surgery, expected to have a nasogastric tube after surgery, renal, hepatic, cardiovascular, metabolic or endocrine dysfunction, history of motion sickness.

Patients belonging to Group-A patient were received Tab Metoclopramide 10mg orally an hour before operation and Group-B were received Tab ondansetron $(0.15 \mathrm{mg} / \mathrm{kg})$ or $8 \mathrm{mg}$ orally an hour before operation,

On arrival of the patients in the operation theatre IV line was inserted and pulse rate, blood pressure and respiratory rate was recorded. Oxygen saturation was measured by pulse oximeter. The patients were pre-oxygenated for three minutes and induction was done with thiopentone $4-5 \mathrm{mg} / \mathrm{kg}$ and fentanyl $1 \mu \mathrm{g} / \mathrm{kg}$, tracheal intubation was facilitated by suxamethonium $2 \mathrm{mg} / \mathrm{kg}$ and general anaesthesia was maintained by halothane $0.5 \%, \mathrm{~N}_{2} 060 \%$ and $\mathrm{O}_{2} 40 \%$. Non-depolarizing muscle relaxant Inj vecuronium $0.1 \mathrm{mg} / \mathrm{kg}$. was used for subsequent muscle relaxation. Intraoperative proper hydration was maintained with Lactated Ringers Solution. At the end of operation, residual effect of muscle relaxant reversed by Inj. Neostigmine $0.04 \mathrm{mg} / \mathrm{kg}$ plus Inj. Atropine $0.02 \mathrm{mg} / \mathrm{kg}$.

Analgesia was provided with per rectal diclofenac suppository (50mg) before extubation. The 24 hours study period was begin upon entry to the recovery room. The number and time of emetic episodes and the number \& time of rescue antiemetic treatments will be recorded. The rescue protocol constituted of metoclopramide $10 \mathrm{mg}$ injected once and repeated once if either nausea or vomiting continued for the next 10 minutes. Patients were carefully observed for any adverse effects like sedation, drowsiness, flushing or any extrapyramidal symptoms.

\section{Statistical Analysis:}

All data were complied and analyzed for statistical significance using unpaired student's t tests or Chi square tests. $\mathrm{P}<0.05$ (CI 95\%) were considered as statistically significant.

\section{Results}

Observations of the present study were analyzed in the light of comparisons among the subject groups (Group-A, Group-B; each group having n=25). All results are expressed as mean $\pm \mathrm{SD}$. The studied groups became statistically matched for age $(p=0.458)$, weight $(p=0.113)$, duration of surgery $(\mathrm{p}=0.907)$. 
Table-I

Demography of studied population

\begin{tabular}{lccc}
\hline Parameter & Group-A & Group-B & \\
& $\mathrm{N}=25$ & $\mathrm{~N}=25$ & P value \\
\hline Age in years & $27.7 \pm 4.39$ & $25.8 \pm 4.66$ & 0.458 \\
Body weight in kg & $56.0 \pm 3.23$ & $53.5 \pm 3.34$ & 0.113 \\
Duration of surgery in min & $30.5 \pm 6.85$ & $30.9 \pm 5.70$ & 0.907 \\
\hline
\end{tabular}

Values are expressed as mean $\pm \mathrm{SD}$. Between groups analysis were done by unpaired student's test (CI=95\%).

Table-II

Changes of heart rate

\begin{tabular}{lcccc}
\hline Group/Time & Base line & At induction & At recovery & Postoperative period \\
\hline Group-A & $84.6 \pm 7.42$ & $93.0 \pm 8.1$ & $109.0 \pm 14.02$ & $89.20 \pm 3.52$ \\
Group-B & $74.3 \pm 6.05$ & $91.0 \pm 9.2$ & $112.0 \pm 12.0$ & $82.0 \pm 7.42$ \\
P-value & 0.016 & 0.62 & 0.761 & 0.009 \\
\hline
\end{tabular}

Values are expressed as mean \pm SEM. Between groups analysis were done by unpaired student's test (CI=95\%).

Table-III

Changes of systolic blood pressure in two groups

\begin{tabular}{lcccc}
\hline Group/Time & Base line & At induction & At recovery & Postoperative period \\
\hline Group-A & $111 \pm 13.7$ & $131 \pm 14.0$ & $131 \pm 13.0$ & $117.5 \pm 7.90$ \\
Group-B & $116 \pm 13.29$ & $128 \pm 12.3$ & $134.0 \pm 21.0$ & $123.5 \pm 14.34$ \\
P-value & 0.44 & 0.341 & 0.561 & 0.29 \\
\hline
\end{tabular}

Values are expressed as mean \pm SEM. Between groups analysis were done by unpaired student's test (CI=95\%).

Variation of heart rate (beats/min) is displayed in Table-II. The baseline heart rate in group-A is $84.6 \pm 7.42$ and in group-B $74.3 \pm 6.05(\mathrm{p}=0.016)$ and postoperative period (group-A: $89.20 \pm 3.52$ and groupB: $82.0 \pm 7.42, \mathrm{p}=0.009)$ significantly varied among the groups. But heart rate variation at induction (in group-A: $93.0 \pm 8.1$, in group-B: $91.0 \pm 9.2, \mathrm{p}=0.62$ ) and at recovery (in group-A $109.0 \pm 14.02$ and in group-B 112.0 $\pm 12.0, \mathrm{p}=0.761$ ) were not significant.

Systolic blood pressure changes were recorded as $\mathrm{mm}$ of $\mathrm{Hg}$ by non invasive automated blood pressure monitor at 5 minutes interval in the intraoperative period and at 30 minutes interval in the post operative period for first 24 hours in the post operative room. The values are presented at four points as per protocol. Systolic blood pressure variation at base line in group-A was $111 \pm 13.7$ and was $116 \pm 13.29$ in group- $B(p=0.44)$, at induction (in group-A $131 \pm 14.0$ and in group-B $128 \pm 12.3$, $\mathrm{p}=0.341$ ), at recovery (in group-A $131 \pm 13$ and in group-B $134 \pm 21.00, \mathrm{p}=0.561$ ) and in postoperative room (in group-A $117.5 \pm 7.9$ in group-B $123.5 \pm 14.34$, $\mathrm{p}=0.29$ ) are not significant between the groups.

Table-IV

Changes of diastolic blood pressure in two groups

\begin{tabular}{lcccc}
\hline Group/Time & Base line & At induction & At recovery & Postoperative period \\
\hline Group-A & $72 \pm 8.86$ & $89.0 \pm 3.99$ & $90.0 \pm 7.36$ & $76.9 \pm 8.19$ \\
Group-B & $68.5 \pm 9.44$ & $87.0 \pm 3.5$ & $91.0 \pm 3.19$ & $75.5 \pm 8.95$ \\
P-value & 0.372 & 0.423 & 0.513 & 0.666 \\
\hline
\end{tabular}

Values are expressed as mean \pm SEM. Between groups analysis was done by unpaired student's test. 
Diastolic blood pressure changes were recorded as $\mathrm{mm}$ of $\mathrm{Hg}$ by non invasive automated blood pressure monitor at 5 minutes interval in the intraoperative period and at 30 minutes interval in the post operative period for first 24 hours in postoperative room. But the values are presented at four points as per protocol. Diastolic blood pressure variation at base line (in group-A: $68.5 \pm 9.44$, in group-B: $72 \pm 8.86, \mathrm{p}=0.372$ ), at induction (in group-A: $87.0 \pm 3.5$, in group-B: $89.0 \pm 3.99, p=0.423)$, at recovery (in group-A: $91.0 \pm 3.19$, in group-B: $90.0 \pm 7.36, \mathrm{p}=0.513$ ) and in post operative room (in group-A: $75.5 \pm 8.95$, in group-B: $76.9 \pm 8.19, \mathrm{p}=0.666$ ) were not significant $(\mathrm{P}>0.05)$ between the groups.

\section{Table-V}

Sedation score in post operative period in two groups

\begin{tabular}{lccc}
\hline Sedation Score & Group-A & Group-B & P-value \\
\hline 0 & $10(40 \%)$ & $20(80 \%)$ & 0.01 \\
1 & $13(52 \%)$ & $3(12 \%)$ & 0.001 \\
2 & $2(8 \%)$ & $2(8 \%)$ & 0 \\
\hline
\end{tabular}

Values are presented as frequency. Within parenthesis are percentages over column total. Analyses were done by chi-square test. Values are regarded as significant if $\mathrm{p}<0.05$ (CI-95\%).

Sedation score after one hour post operatively varies from 0 to 2 for both the groups. In group-B, it was 0 for $80 \%, 1$ for $12 \%$, and 2 for $8 \%$ patients respectively, In group-A, it was 0 for $40 \%, 1$ for $52 \%$ and 2 for $8 \%$ patients respectively and was statistically significant $(p<0.05)$.

\section{Table-VI}

Nausea and Vomiting in post operative period in two groups

\begin{tabular}{lccc}
\hline Variable & Group-A & Group-B & P-value \\
\hline Nausea & $20(80 \%)$ & $6(24 \%)$ & 0.001 \\
Vomiting & $16(64 \%)$ & $4(16 \%)$ & 0.001 \\
\hline
\end{tabular}

Values are presented as frequency. Within parenthesis are percentages over column total. Analyses were done by chi-square test. Values are regarded as significant if $\mathrm{p}<0.05$ (CI-95\%).

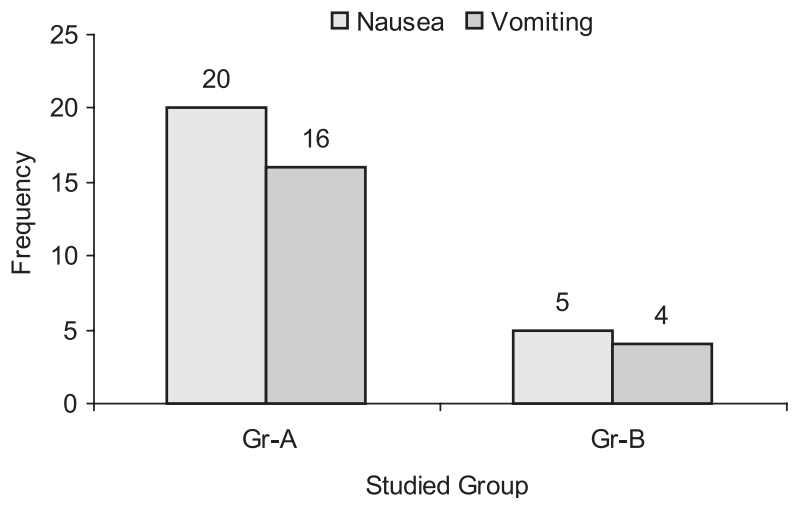

Fig.-1: Frequency of nausea and vomiting in two groups

The incidence of nausea was $24 \%$ in Group-B, $80 \%$ in Group-A ( $p=0.001)$ and vomiting was $16 \%$ in Group-B, $64 \%$ in Group-A ( $p=0.001)$. The difference among the groups was statistically significant.

There was no complication like flushing or any extrapyramidal symptoms in both the groups.

\section{Discussion}

Nausea and vomiting are common postoperative complaints and some times dangerous side effects following surgery under anaesthesia. Most of the incidents of nausea and vomiting occur during the first two hours of recovery from anaesthesia.

The aetiology of PONV is multifactorial. Factors associated with an increased risk of postoperative emesis include age, gender, obesity, previous history of motion sickness or postoperative vomiting, anxiety, gastroparesis, pain, hypoxia, type of anaesthetic, hypotension and type and duration of the surgical procedure. In the present study concern factors are type of anaesthesia, female patient and type of surgery (gynaecological laparoscopic surgery). Incidence of nausea and vomiting is two to three times more in female due to changing endocrine environment, which sensitize the brain stem emetic mechanism. During laparoscopic surgery the effect of pneumoperitonium as well as some traction of vagal innervated gut may play role in triggering emesis. So patients undergoing gynaecological laparoscopy assisted surgery are at high risk for postoperative nausea and vomiting.

The antiemetics are now mainstay of therapy to prevent nausea and vomiting. The introduction of $5-\mathrm{HT}_{3}$ receptor antagonist in 1990s was heralded 
as a major advance in the treatment of PONV because of less adverse effects that were observed with commonly used traditional antiemetic.

Malins et al. have also studied the efficacy of ondansetron compared with metoclopramide. ${ }^{10}$ One hundred and fifty patients were given oral premedication 1 hour before gynaecological laparoscopy with ondansetron mg, metoclopramide $10 \mathrm{mg}$ or placebo. There was a significantly greater number of asymptomatic patients in the ondansetron group in the 48 hours after operation compared with the other two groups ( $74 \%, 58 \%$ and $50 \%$, respectively $(p<0.05)$. The average duration of anaesthesia was 21 minutes. The number of patients receiving rescue anti-emetic treatment was $38 \%$, $57 \%$ and $68 \%$ respectively. There was no significant difference between the groups with respect to heart rate, systolic and diastolic blood pressure.

In our study, incidence of nausea and vomiting in group-A (those received ondansetron) were $24 \%$ and $16 \%$ and in group-B (Those received metoclopramide) were $80 \%$ and $64 \%$ that means asymptomatic patients in group-A is $76 \%$ in comparison to group$B$ is $20 \%(p=0.001)$. The average duration of anaesthesia was 30 minutes. In our study no patients received rescue anti-emetic treatment because there was no intractable vomiting (vomiting those occurred was 1 to 2 times only). Heart rate differences among the groups at base line $(p=0.016)$ and postoperative period $(\mathrm{p}=0.009)$ was significant Systolic and diastolic blood pressure differences among the groups were not significant $(\mathrm{p}<0.05)$.

The difference in the results of asymptomatic patients in our study $20 \%$ compared with those of Malins et al. $58 \%$, may be explained by; a small number of population and the mean duration of anaesthesia was greater in our study. Heart rate variation among the groups at base line and in post operative period were significant, may be explained by, a small number of patients.

Sedation score after one hour post operatively varies from 0 to 2 for both the groups. In group-A, it was 0 for $80 \%, 1$ for $12 \%$, and 2 for $8 \%$ patients respectively, In group-B, it was 0 for $40 \%, 1$ for $52 \%$ and 2 for $8 \%$ patient respectively and was statistically significant $(p<0.05)$. It may be explained that patients of Group$B$ was more sedated.

\section{Conclusion}

Under the condition of the present study we found that oral premedication with ondansetron $8 \mathrm{mg}$ is more effective and produce fewer side effects than metoclopramide $10 \mathrm{mg}$ in preventing postoperative nausea and vomiting after laparoscopy assisted gynaecological diagnostic procedure.

\section{References:}

1. Aitkendhead AR. Postoperative care. In: Alan R. Aitkenhead, David J. Rowbotham, Graham Smith, eds. Textbook of Anaesthesia. $4^{\text {th }}$ Edn. Churchill Livingstone, London, 2001:541

2. Kapur PA. The big, little problem. Anaesth Anal 1991; 73:243-5

3. Andrews PLR. Physiology of nausea and vomiting. Br J Anaesth 1992; 69:2-19

4. Hernandez Conte AT. Postoperative nausea and vomiting: a review of antiemetic pharmacological interventions. Anesth Pharmacol Physiol Rev 1996;4:57-65

5. Watcha MF, White PF. Postoperative nausea and vomiting: Its etiology, treatment and prevention. Anesthesiology 1992;77:162-84.

6. Rowbotham DJ Current management of postoperative nausea and vomiting. $\mathrm{Br} J$ Anaesth 1992: 69(1):46-59.

7. Hindle AT. Recent developments in the physiology and pharmacology of 5-hydroxytryptamine. Br J Anaesth 1994; 73: 395-407

8. Seynaeve C, Verwij J, DeMulder PHM. 5- $\mathrm{HT}_{3}$ receptor antagonists- a new approach in emesis: a review of ondansetron, granisetron and tropisetron. Anti-Cancer Drugs 1991; 2:343-355

9. Adjuncts to Anesthesia. In: G. Edward Morgan, Jr. Maged S. Mikhail Michael J. Murray with Philip Larson Jr. Clinical Anesthesiology, $3^{\text {rd }}$ Edn, Mc Graw Hill, New York, 2002 : 245-248

10. Malin AF, Field JM, Nesling PM, Copper GM. Nausea and vomiting after gynaecological laparoscopy: comparison of premedication with oral ondansetron, metoclopramide and placebo. Br J Anaesth 1994; 72:231-3. 